\title{
Ageing of the couplings between cardiac, respiratory and myogenic activity in humans*
}

\author{
Valentina Ticcinelli ${ }^{1}$, Tomislav Stankovski ${ }^{1}$, Peter V. E. McClintock ${ }^{1}$ and Aneta Stefanovska ${ }^{1}$
}

\begin{abstract}
The balance and functionality of the cardiovascular system are maintained by a network of couplings between the different oscillations involved. We study the effect of ageing on these interactions through the application of wavelet analysis, and by the use of dynamical Bayesian inference to compute coupling functions. The method, applied to phases extracted from microvascular flow recorded by laser Doppler flowmetry (LDF), reveals the coupling functions between oscillations propagated to the smallest vessels. Consistent with earlier work based on analysis of cardiac and respiratory phases obtained from direct measurements, our analysis demonstrates an impairment of the propagated cardio-respiratory coupling with ageing. The coupling weakens despite the increased cardiac component in the LDF with ageing. Our results bring new insight to the effect of ageing on cardiovascular regulation that might help improve the diagnostic potential of LDF monitors.
\end{abstract}

\section{INTRODUCTION}

Oscillations are ubiquitous in nature. Light travels in waves, with different frequencies that we perceive as colours. Sound propagates as oscillations too, differentiated by amplitude and frequency. We can take delight in the most beautiful landscape or the most pleasant music just because they manifest themselves to us as ensembles of waves. For the human body, oscillations not only provide a means of interaction with the surroundings, but also the basis of inner functional mechanisms [1], [2]. Countless simultaneous oscillatory processes occur in each individual, continuously, from conception until death. Life is nothing but change, and the way oscillations generate and interact within our body while we age is no exception to this rule.

In this paper we discuss oscillatory interactions and their spatial propagation within the cardiovascular system. Based on wavelet analysis and computation of coupling functions between cardiac and respiratory oscillations, we show that these mechanisms become weaker with age.

\section{CARDIOVASCULAR OSCILLATIONS AND THEIR PROPAGATION WITHIN THE BLOOD FLOW}

Far from being a system of rigid tubes, the arteries, veins and capillaries of the vascular network are elastic, and not only in a passive way: they are living entities, with their own specific contractile properties [3]-[6]. The oscillations propagating within the circulatory system from

\footnotetext{
*The work was supported by the Engineering and Physical Sciences Research Council, United Kingdom (Grant No. EP/100999X1), by the Joint Research Councils' New Dynamics of Ageing programme administered by the Economic and Social Research Council, United Kingdom (Grant No. RES-356-25-0006), and by the Slovenian Research Agency

${ }^{1}$ Physics Department, Lancaster University, LA1 4YW, UK aneta@lancaster.ac.uk
}

central origins are therefore not identical with their sources, but are locally modulated by complex mechanisms operating in the layers of cells forming the vessels' walls.

\section{A. Cardiac oscillations}

The most evident source of physiological oscillations is the heart. The oscillations that enable it to fulfil its central role of pumping the blood through the circulatory system are self-sustained, but are not independent of other processes occurring in the organism. Interactions between oscillations keep the system stable and well-coordinated: the frequency and strength of the heartbeat are strongly modulated by a demand/supply mechanism [7]. In healthy subjects, the activity of the heart propagates down to the peripheral capillary level and is clearly distinguishable in a signal recorded on the skin by laser-Doppler flowmetry (LDF).

\section{B. Respiration}

The respiratory expansions and contractions of the lungs generate an oscillatory pressure in the network of vessels [8]: the mechanism of its propagation in the blood flow is more subtle than that of cardiac oscillations, and the shape of a respiratory peak in the power spectra of LDF signals is more subject/location-sensitive. Moreover, the respiratory activity modulates over the heart, accelerating and decelerating its rhythm every time a breath is taken, via the coupling known as respiratory sinus arrhythmia [9].

\section{Myogenic activity}

A vascular oscillation centred around $0.1 \mathrm{~Hz}$ has been detected by several studies in the past [6], [10], [11]. The attribution of a source to this oscillation is still controversial. Some authors discuss a sympathetic origin of these oscillations in the microvascular flow [12], [13] and, in relation to heart rate variability, have associated them with baroreceptor activity. However, the myogenic theory suggests that the motion is caused directly by pacemaker cells in arterial smooth walls [3], [11]: these cells have the ability to contract in response to the variation of intravascular pressure [3]. Because the sympathetic system innervates the vascular myocytes too, the myogenic and sympathetic systems are coupled, so that it is not easy to isolate and study their specific contributions. However, studies on anæsthesia and skinflaps were able to uncouple the systems and distinguish the myogenic $(0.1 \mathrm{~Hz})$ from the slower sympathetic oscillation $(0.04 \mathrm{~Hz})$ [11]. The studies that supported the sympathetic hypothesis may perhaps have been biased by the use of the Fourier transform, which lacks the resolution needed to distinguish neighbouring low-frequency oscillations. 


\section{Slower oscillations}

Three additional characteristic oscillations have been detected in the lower-frequency region of the power spectra of blood flow [1]. They have been attributed to sympathetic (0.021-0.052 Hz), nitric-oxide-dependent endothelial $(0.0095-0.021 \mathrm{~Hz})$ and nitric-oxide-independent endothelial $(0.005-0.0095 \mathrm{~Hz})$ processes. Although the features of the power spectra of these slow oscillations can be observed by use of the wavelet transform (sec. V-A), the investigation of their phase-dynamics is more arduous. Certain conditions need to be fulfilled in order to be able to rely on the meaningfulness of phases reconstructed from real data. The most important of these is the assumption that the spatially-distributed oscillators generating the signal are fully synchronised on the macroscopic level. In practice, this hypothesis is never perfectly fulfilled, but the approximation is compensated for by the large number of periods recorded. The length of the LDF signals sets a practical limitation to this compromise; hence in this paper the investigation has been limited to the faster three oscillations (table I).

TABLE I

SUMMARY OF THE ANALYSED OSCILLATIONS

\begin{tabular}{|l|l|l|l|}
\hline $\begin{array}{l}\text { band } \\
\text { number }\end{array}$ & $\begin{array}{l}\text { oscillation } \\
\text { source }\end{array}$ & $\begin{array}{l}\text { central } \\
\text { frequency }\end{array}$ & $\begin{array}{l}\text { frequency } \\
\text { interval }\end{array}$ \\
\hline I & cardiac & $1 \mathrm{~Hz}$ & $2-0.6 \mathrm{~Hz}$ \\
II & respiratory & $0.3 \mathrm{~Hz}$ & $0.6-0.145 \mathrm{~Hz}$ \\
III & myogenic & $0.1 \mathrm{~Hz}$ & $0.145-0.052 \mathrm{~Hz}$ \\
\hline
\end{tabular}

\section{COUPLED OSCILLATORS}

The cardiovascular system can be modeled as a network of coupled oscillators [1]. The inverse problem of evaluating the mutual interactions, starting from a real signal, has been tackled mainly through correlation-based approaches, e.g. the wavelet bispectrum [14]. This kind of computation allows one to evaluate the existence and strength of a coupling, but does not easily provide information about the directionality of the interaction, or its mechanisms.

\section{Methods}

\section{A. Wavelet analysis}

The signals considered can be explored in the timefrequency domain by means of the wavelet transform (WT) [6]. The existence of characteristic time-persistent ridges in the transform implies oscillatory processes of deterministic nature; and their appearance at the same frequencies in different signals suggests their common origin. By yielding values of amplitude and phase for each time/frequency combination, the (WT) allows one to compare time-averaged power spectra and calculate the phase coherence between different signals. The Morlet mother wavelet is used, providing a direct relationship between the scale and frequency. The adaptive scaling (adjustable window) provides optimal frequency resolution (e.g. logarithmic) for the investigation of the broad frequency range typical of cardiovascular oscillations.

\section{B. Decomposition method}

Nonlinear mode decomposition (NMD) [15] has been applied to extract and separate the different oscillations from the signals. The method was particularly well-suited to this situation as it allows to extract a mode with the help of a reference signal : thus, oscillations I and II were extracted from the LDF signal by using the ECG and respiration signals as references. Moreover, the method is noise robust, and provides the instantaneous phase.

\section{Coupling functions}

Recently, a novel technique has been developed for the investigation of coupling dynamics [16]-[18]. The interactions are modeled with a network of coupled phase oscillators: by the application of the dynamical Bayesian inference [19]-[22] to the network, it is possible to compute the set of parameters regulating the mutual influences, and to separate out the noise [19]. Tridimensional visualisation of each coupling function $(\mathrm{CF})$ can be used to provide deeper insight into individual interactions by showing the evolution of a coupling parameter as a function of two phases. The amplitude and shape of this relationship reveals not only the strength but also the directionality and the functional mechanism that regulates the coupling [16]-[18].

\section{THE EFFECT OF AGEING}

Signals from two groups of subjects were analysed: 29 (14F) young healthy (aged $24.4 \pm 3.4$ years), and $22(13 \mathrm{~F})$ aged healthy $(71.1 \pm 6.6$ years). The study was approved by the Lancaster University Research Ethics Committee. The electrical activity of the heart was recorded by electrocardiography (ECG). The respiration signal was acquired using a stretch-sensitive band wrapped around the thorax, and the blood flow on the forearm by using LDF. Signals were recorded for 30 minutes, with subjects supine in a comfortable setting in a room at constant temperature. See [23], [24] for further details.

Statistical analysis was performed using a non-parametric two-sided Wilcoxon rank sum test and $p<0.05$ was considered as significant.

\section{A. Wavelet transform}

Typical WTs of LDF signals for young and aged subjects are shown in figs. 1-a and 1-b respectively. The timecontinuity of activity III, together with I, can be observed in the time-frequency space of the young subject's LDF signal (fig. 1-a). For the aged subject (fig. 1-b), component I is relatively much stronger than the lower frequency oscillations.

\section{B. Power spectra}

Blood flow power appears higher overall in the aged group (fig. 2): the age-related decrease in skin thickness [25] may affect this measure, resulting in a stronger LDF signal. However, skin properties cannot affect the distribution of the power, and the strikingly significant difference in component I may be due to the effect of age on the vascular tissue. The 
(a)
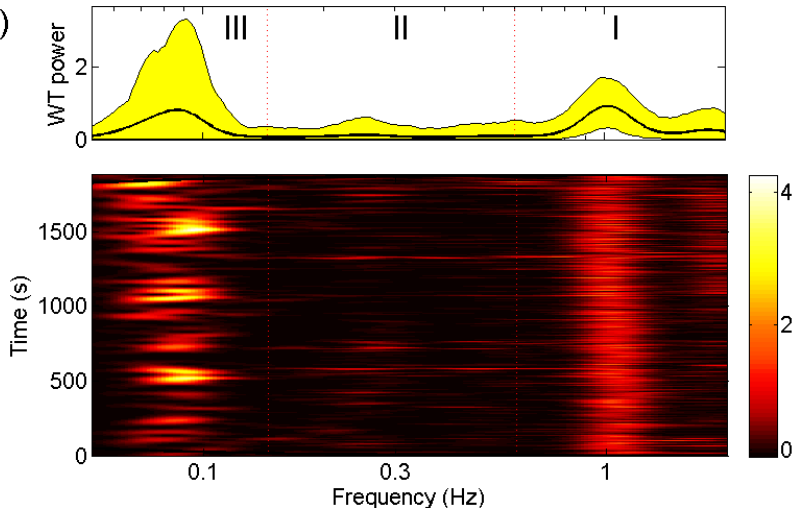

(b)
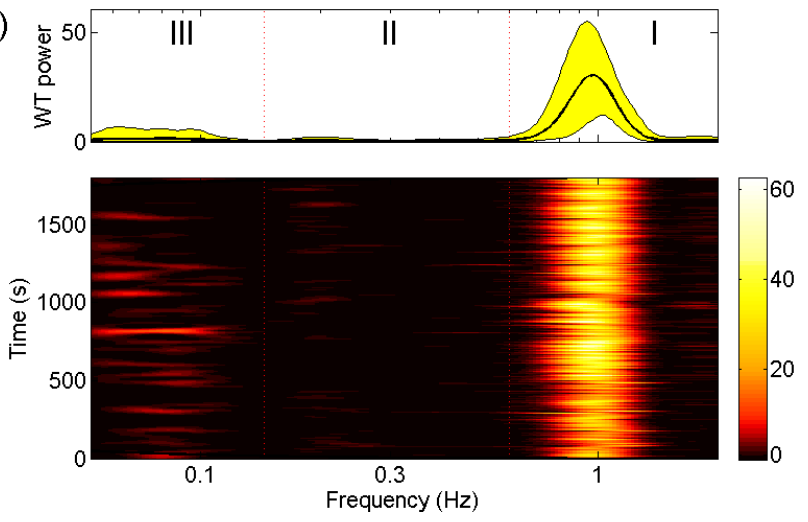

Fig. 1. Typical wavelet power and time-averaged spectrum of the LFD signal for (a) a young subject and (b) an aged subject.

increased vessels' radius and decreased vessels' elasticity [26] may have led to decreased local active oscillations, and to stronger direct propagation of the centrally generated waves (i.e. I and II).

\section{Wavelet phase coherence}

The coordination between the oscillatory activity in the source signal and the propagated oscillation in the LDF can be measured with wavelet phase coherence [27]. In this case, optical permeability of the skin does not affect the result, as the phase of the oscillation is unrelated to its amplitude.

With ageing, intervals of significantly higher timeaveraged wavelet phase coherence can be found within band I, together with a window of significantly lower myogenic coherence (fig. 3). This evidence confirms the suggestion proposed above in the case of the power (sec. V-B), i.e. that with ageing the active response of the vessels' cells decreases. This, joined with the general loss of elastic properties, results in a more direct propagation of the oscillations in interval I.

\section{Coupling functions}

After extraction of the modes and phases $\left(\phi_{r}, \phi_{c}\right)$ from the signals by application of NMD [15], the cardio-respiratory interaction and its effect on blood flow have been studied by the means of CFs. Fig. 4 shows the averaged cardiorespiratory CFs for young (fig. 4a,d) and aged subjects (fig. $4 \mathrm{~b}, \mathrm{e})$ and inter-group-shuffled surrogates [28] (fig. 4 c,f). In

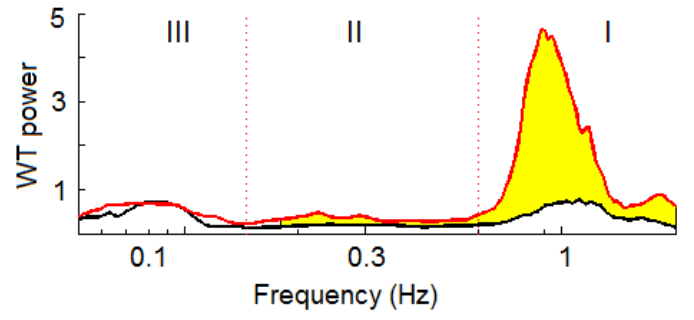

Fig. 2. Time-averaged median wavelet power for young (black) and aged subjects (red). Significant differences are highlighted with yellow

the figure, $q_{C}$ indicates the coupling function between the central sources of oscillations, i.e. I and II extracted from the ECG and respiration signals, while $q_{L}$ indicates the coupling function between locally propagated oscillations, i.e. I and II extracted from LDF signals.

For young subjects, $q_{C}$ (fig. 4-a) the form of the CF resembles a sinusoidal wave propagating along the respiration phase axis (while along the cardiac direction it remains constant): this topography indicates that the frequency of the heart undergoes a modulation coming from each respiratory period. The shift of this sinusoidal wave suggests that the heart is speeded up during inhalation and slowed down in the middle of the breathing cycle (around $\pi$ ). The shape of this relationship is preserved in the time-averaged CF from the aged subjects (fig. 4-b), even if there is a clear decrease of the amplitude, resulting from a less deterministic shape of the $\mathrm{CF}$ among different subjects. Comparison to the surrogates shows that the $\mathrm{CF}$ is still relevant here. Therefore, it can be concluded that the mechanism of respiratory arrythmia is affected by ageing, becoming weaker and more variable, even if still present. The results reported in fig. 4-a and fig. 4$\mathrm{b}$ confirm what was found earlier for cardio-respiratory $\mathrm{CFs}$ by Iatsenko et al. [24].

With regard to the manifestation of the coupling in the LDF signal, it can be seen that for young subjects (fig. 4-d) the form of $q_{L}$ still resembles the sinusoidal shape of fig. 4-a. The shift observed in the figure could be due to the delayed arrival of the propagating respiration wave in the blood flow. It is noteworthy that, although the varied shape of this $\mathrm{CF}$ for different subjects results in an averaged CF of

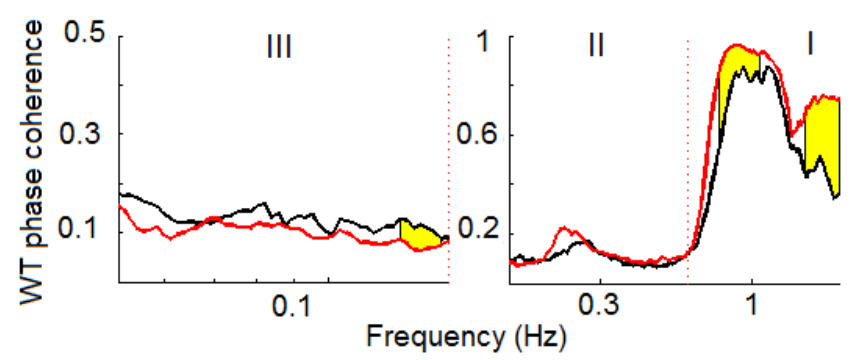

Fig. 3. Median wavelet phase coherence between ECG signal and LDF signal for young subjects (black line) and aged subjects (red line). Significant differences in coherence were checked for each frequency and are highlighted in yellow. Significant coherence was observed in frequency interval I, corresponding to the cardiac frequency and its first harmonic. 

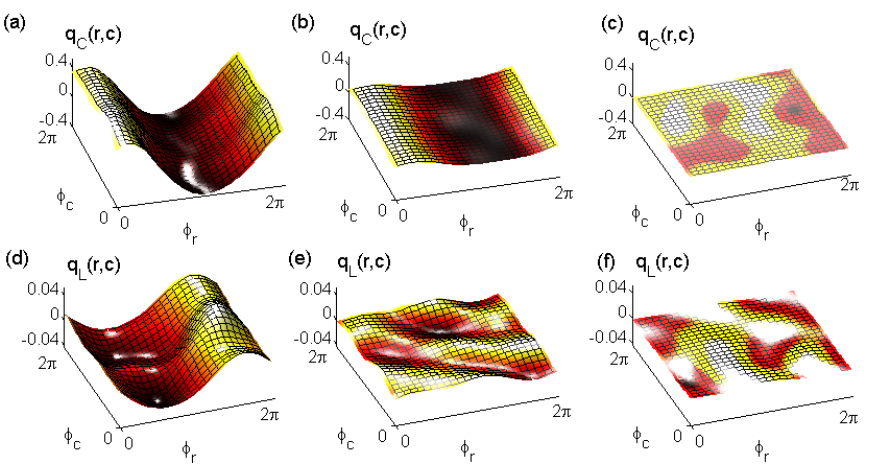

Fig. 4. Averaged CFs of the phases $\phi_{c}$ and $\phi_{r}$ of cardiac and respiratory activity over the cardiac frequency for $(\mathrm{a}, \mathrm{d})$ young, $(\mathrm{b}, \mathrm{e})$ aged subjects and $(\mathrm{c}, \mathrm{f})$ inter-groups surrogates. $(\mathrm{a}, \mathrm{b}, \mathrm{c}) q_{C}$ shows the coupling detected from the central sources (ECG and respiration), while (d,e,f) $q_{L}$ shows the coupling propagated into the LDF signal.

smaller amplitude, the morphological features described for fig. 4-a survive the averaging procedure. For older subjects, the average $q_{L}$ loses its sinusoidal characteristic, becoming comparable to surrogates of fig. 4-f. This could suggest that the coupling, already weakened at source, is in addition attenuated by the decreased reactivity of aged vessels.

\section{CONCLUSIONS}

The outcomes of this investigation are consistent with what has been reported earlier [23], [24], i.e. that ageing compromises the delicate network of interactions regulating the cardio-respiratory-vascular system, on several levels. The active inter-modulation between oscillations appears to be weakened in aged subjects both locally (sec. V-A, sec. V-B, sec. V-C) and systemically (sec. V-D). Moreover, a weaker cardio-respiratory coupling as propagated to the microvascular blood flow has been found for the aged group. Gender differences may have existed but the numbers of subjects studied would not allow any firm conclusions to be drawn.

\section{ACKNOWLEDGMENT}

We are grateful to Dmytro Iatsenko for valuable discussions, to Alan Bernjak and Yuri Shiogai for the data acquisition, and to Jane Owen-Lynch and Peter Clarkson for the biological and clinical insights.

\section{REFERENCES}

[1] A. Stefanovska, "Coupled oscillators: Complex but not complicated cardiovascular and brain interactions," IEEE Eng. Med. Bio. Magazine, vol. 26, no. 6, pp. 25-29, 2007.

[2] A. Stefanovska and M. Bračič, "Physics of the human cardiovascular system," Contemp. Phys., vol. 40, no. 1, pp. 31-55, 1999.

[3] P. C. Johnson, "The myogenic response," News Physiol. Sci., vol. 6, pp. 41-42, 1991.

[4] S. Bertuglia, A. Colantuoni, and M. Intaglietta, "Effects of L-NMMA and indomethacin on arteriolar vasomotion in skeletal-muscle microcirculation of conscious and anesthetized hamsters," Microvasc. Res., vol. 48, no. 1, pp. 68-84, 1994.

[5] J. Karstrup, J. Bühlow, and N. A. Lassen, "Vasomotion in human-skin before and after local heating recorded with laser Doppler flowmetry A method for induction of vasomotion," Int. J. Microcirc.: Clin. Exp., vol. 8, no. 2, pp. 205-215, 1989.
[6] A. Stefanovska, M. Bračič, and H. D. Kvernmo, "Wavelet analysis of oscillations in the peripheral blood circulation measured by laser Doppler technique," IEEE Trans. Bio. Med. Eng., vol. 46, no. 10, pp. 1230-1239, 1999.

[7] J. P. Saul, R. D. Berger, P. Albrecht, S. P. Stein., M. H. Chen, and R. J. Cohen, "Transfer function analysis of the circulation - unique insights into cardiovascular regulation," Am. J. Physiol., vol. 261, no. 4, pp. H1231-H1245, 1991.

[8] D. L. Eckberg, "The human respiratory gate," J. Physiol. (London), vol. 548, no. 2, pp. 339-352, 2003.

[9] M. Clynes, "Respiratory sinus arrhythmia: laws derived from computer simulation," J. Appl. Physiol., vol. 15, no. 5, pp. 863-874, 1960.

[10] A. Stefanovska, M. Bračič Lotrič, S. Strle, and H. Haken, "The cardiovascular system as coupled oscillators?," Physiol. Meas., vol. 22, no. 3, pp. 535-550, 2001.

[11] T. Söderström, A. Stefanovska, M. Veber, and H. Svenson, "Involvement of sympathetic nerve activity in skin blood flow oscillations in humans," Am. J. Physiol.: Heart. Circ. Physiol., vol. 284, no. 5, pp. H1638-H1646, 2003.

[12] H. M. Stauss, E. A. Anderson, W. G. Haynes, and K. C. Kregel, "Frequency response characteristics of sympathetically mediated vasomotor waves in humans," Am. J. Physiol.: Heart. Circ. Physiol., vol. 274, no. 4, pp. H1277-H1283, 1998.

[13] A. Cevese, G. Gulli, E. Polati, L. Gottin, and R. Grasso, "Baroreflex and oscillation of heart period at $0.1 \mathrm{~Hz}$ studied by $\alpha$-blockade and cross-spectral analysis in healthy humans," J. Physiol. (London), vol. 531, no. 1, pp. 235-244, 2001.

[14] J. Jamšek, M. Paluš, and A. Stefanovska, "Detecting couplings between interacting oscillators with time-varying basic frequencies: Instantaneous wavelet bispectrum and information theoretic approach," Phys. Rev. E, vol. 81, no. 3, p. 036207, 2010.

[15] D. Iatsenko, A. Stefanovska, and P. V. E. McClintock, "Nonlinear mode decomposition: a noise-robust, adaptive, decomposition method," Phys. Rev. E., under revision, arXiv:1207.5567, 2015.

[16] B. Kralemann, A. Pikovsky, and M. Rosenblum, "Reconstructing phase dynamics of oscillator networks," Chaos, vol. 21, p. 025104, 2011.

[17] B. Kralemann, A. Pikovsky, and M. Rosenblum, "Reconstructing effective phase connectivity of oscillator networks from observations," New J. Phys., vol. 16, no. 8, p. 085013, 2014.

[18] T. Stankovski, V. Ticcinelli, P. V. E. McClintock, and A. Stefanovska, "Coupling functions in networks of oscillators," New J. Phys., vol. 17, no. 3, p. 035002, 2015.

[19] T. Stankovski, A. Duggento, P. V. E. McClintock, and A. Stefanovska, "Inference of time-evolving coupled dynamical systems in the presence of noise," Phys. Rev. Lett., vol. 109, p. 024101, 2012.

[20] U. von Toussaint, "Bayesian inference in physics," Rev. Mod. Phys., vol. 83, no. 3, pp. 943-999, 2011.

[21] J. Daunizeau, K. J. Friston, and S. J. Kiebel, "Variational Bayesian identification and prediction of stochastic nonlinear dynamic causal models," Physica D, vol. 238, no. 21, pp. 2089-2118, 2009.

[22] W. D. Penny, V. Litvak, L. Fuentemilla, E. Duzel, and K. Friston, "Dynamic causal models for phase coupling," J. Neurosci. Meth., vol. 183, no. 1, pp. 19-30, 2009.

[23] Y. Shiogai, A. Stefanovska, and P. V. E. McClintock, "Nonlinear dynamics of cardiovascular ageing," Phys. Rep., vol. 488, pp. 51-110, 2010.

[24] D. Iatsenko, A. Bernjak, T. Stankovski, Y. Shiogai, P. J. OwenLynch, P. B. M. Clarkson, P. V. E. McClintock, and A. Stefanovska, "Evolution of cardio-respiratory interactions with age," Phil. Trans. R. Soc. Lond. A, vol. 371, no. 1997, p. 20110622, 2013.

[25] N. A. Fenske and C. W. Lober, "Structural and functional changes of normal aging skin," J. Amer. Acad. Dermatol., vol. 15, no. 4,1, pp. 571-585, 1986.

[26] R. I. Kelly, R. Pearse, R. H. Bull, J.-L. Leveque, J. de Rigal, and P. S. Mortimer, "The effects of aging on the cutaneous microvasculature," J. Am. Acad. Dermatol., vol. 33, no. 5, pp. 749-756, 1995.

[27] L. W. Sheppard, A. Stefanovska, and P. V. E. McClintock, "Testing for time-localised coherence in bivariate data," Phys. Rev. E, vol. 85, p. 046205, 2012.

[28] T. Schreiber and A. Schmitz, "Improved surrogate data for nonlinearity tests," Phys. Rev. Lett., vol. 77, no. 4, pp. 635-638, 1996. 